\title{
Biometría de los Músculos Extensor Corto de los Dedos y Extensor Corto del Hallux en Individuos Brasileños: Análisis Anatómico Para su uso Como Colgajo Muscular en Cirugías Reconstructivas
}

\author{
Biometry of the Extensor Digitorum Brevis and Extensor Hallucis Brevis Muscles in Brazilian \\ Individuals: Anatomical Analysis For Use as a Muscle Flap in Reconstructive Surgeries
}

Caamaño-Binimelis, C. ${ }^{1,2}$; Riveros, A. ${ }^{1,3}$; Sousa-Rodrigues, C.4; Olave, E. ${ }^{5}$

CAAMAÑO-BINIMELIS, C.; RIVEROS, A.; SOUSA-RODRIGUES, C. \& OLAVE, E. Biometría de los músculos extensor corto de los dedos y extensor corto del hallux en individuos brasileños: análisis anatómico para su uso como colgajo muscular en cirugías reconstructivas. Int. J. Morphol., 38(4):1010-1017, 2020.

RESUMEN: El músculo extensor corto de los dedos (ECD) se sitúa junto con el músculo extensor corto del hallux (ECH) en la región dorsal del pie y están encargados de colaborar con la acción agonista de los músculos extensor largo de los dedos (ELD) y extensor largo del hallux (ELH), en la extensión de los cuatro dedos mediales en las articulaciones metatarsofalángicas. Esta condición complementaria permite su transferencia quirúrgica hacia otras regiones receptoras sin afectar la funcionalidad extensora de los dedos del pie motivo por el cual durante las últimas décadas se ha convertido en un tejido importante para la reconstrucción de lesiones tisulares, sin embargo, son escasos los estudios biométricos que lo describan en detalle. El objetivo de esta investigación fue establecer la longitud, ancho y área de extensión de estos músculos acompañada de la determinación de las distancias de éstos respecto a la cuña medial y la base del quinto metatarsiano para su uso quirúrgico. Sumado a lo anterior y posterior a la descripción de las variantes musculares encontradas se determinó los puntos motores de inervación de estos dos músculos. Para ello se estudiaron 36 miembros inferiores formolizados de individuos adultos brasileños pertenecientes a la Universidad Federal de Alagoas (UFAL), Maceió, Brasil. Las mediciones se realizaron con un cáliper marca Mitutoyo de $0,01 \mathrm{~mm}$ de precisión obteniendo una longitud, ancho y área de $6,89 \pm$ $1,64 \mathrm{~cm}, 3,81 \pm 0,42 \mathrm{~cm}$ y $31,98 \pm 7,60 \mathrm{~cm}^{2}$ en el lado derecho de $6,91 \pm 1,64 \mathrm{~cm}, 3,68 \pm 0,46 \mathrm{~cm}$ y $30,75 \pm 7,61 \mathrm{~cm}^{2}$ en el izquierdo respectivamente. En el $17 \%$ de los casos hay presencia de tendones accesorios para el músculo ECD. La distancia desde el margen medial del músculo ECH respecto a la cuña medial y del margen lateral del músculo ECD a la base del quinto metatarsiano fue de 1,97 $\pm 0,43$ y $1,72 \pm 0,41$ al lado derecho y de 2,01 $\pm 0,62$ y $1,87 \pm 0,36$ al lado izquierdo respectivamente. Los puntos motores (Pm) predominaron en un $64 \%$ en el tercio medio del músculo ECH y en un $64 \%$ en el tercio proximal del músculo ECD. Estos resultados son un aporte significativo, tanto para quienes realizan cirugía ortopédica como para el conocimiento detallado de la anatomía dorsal del pie.

PALABRAS CLAVE: Anatomía, Dorso del pie, Inervación, músculo extensor corto de los dedos, Músculo extensor corto del Hallux, Puntos motores, Biometría.

\section{INTRODUCCIÓN}

El músculo extensor corto de los dedos (ECD) es un músculo delgado que surge de la superficie superolateral del calcáneo, el ligamento talocalcáneo interóseo y de la superficie profunda del origen del retináculo extensor. Se inclina distal y medialmente a través del dorso del pie y termina en cuatro tendones que se insertan en el margen lateral de los tendones de músculo extensor largo de los dedos (ELD) (Standring, 2016). El vientre muscular medial de este músculo termina en un tendón que cruza la arteria dorsal del pie superficialmente para insertarse en la cara dorsal de la base de la falange proximal del hallux; a este vientre se le denominó músculo extensor corto del hallux (ECH) pudiendo sus fibras estar completamente separadas o fusionadas con las fibras del ECD (Macalister, 1875), considerando que los músculos ECD y ECH no son agonistas, sino que cumplen una labor complementaria en la extensión

\footnotetext{
${ }^{1}$ Departamento de Ciencias Morfológicas, Facultad de Medicina y Ciencia, Universidad San Sebastián, Lientur 1457, Concepción 4080871, Chile.

${ }^{2}$ Programa de Magíster en Ciencias, mención Morfología, Universidad de La Frontera, Temuco, Chile.

${ }^{3}$ Programa de Doctorado en Ciencias Morfológicas, Universidad de La Frontera, Chile.

${ }^{4}$ Universidad Federal de Ciencias da Saúde de Alagoas (UFAL), Maceió, Brasil.

${ }^{5}$ Facultad de Medicina, Universidad de La Frontera, Temuco, Chile.
} 
CAAMAÑO-BINIMELIS, C.; RIVEROS, A.; SOUSA-RODRIGUES, C. \& OLAVE, E. Biometría de los músculos extensor corto de los dedos y extensor corto del hallux en individuos brasileños: análisis anatómico para su uso como colgajo muscular en cirugías reconstructivas. Int. J. Morphol., 38(4):1010-1017, 2020.

de las articulaciones metatarsofalángicas realizada por los músculos ELD y extensor largo del hallux (ELH). McCraw \& Furlow (1975) señalaron que existe una fuerte evidencia que sugiere que la extracción de los músculos ECD y ECH no provoca déficit clínico significativo, razón por la cual estos músculos se consideran candidatos habituales en el desarrollo de procedimientos quirúrgicos que requieran la utilización del colgajo dorsal del pie y que no generen un deterioro en la extensión de los dedos (Chattar-Cora \& Pederson, 2006).

Considerando que estos músculos son candidatos importantes para las cirugías reconstructivas, el conocer las principales variantes anatómicas resulta un factor importante. Al respecto, el músculo ECD con frecuencia presenta variantes anatómicas en el número de sus cabezas que pueden variar de dos a cinco vientres musculares (Bergman et al., 1988; Sarrafian \& Kelikian, 2011). Sirasanagandla et al. (2013) encontraron en un $9 \%$ de su muestra, la presencia de tres cabezas dirigidas a los tres dedos mediales, lo que se sumó a la ausencia del tendón para el cuarto dedo y la presencia de un tendón accesorio para el primer músculo interóseo dorsal.

Estos hallazgos concuerdan con las variantes presentadas por Macalister, quien describió proyecciones de la segunda cabeza hacia el primer músculo interóseo dorsal o de la tercera cabeza hacia el segundo músculo interóseo dorsal. En esta misma serie de 44 casos, Sirasanagandla et al. encontraron que en un $7 \%$, las cuatro cabezas del músculo ECD presentaron un tendón adicional originados desde la segunda cabeza equivalentes al $75 \%$ de la muestra; en el otro $25 \%$ de los casos lo hizo la 3era cabeza concordando con las variantes descritas por Sarrafian \& Kelikian. Además, el mismo autor reportó que en un solo miembro se presentaron seis tendones los cuales tuvieron su origen en la segunda cabeza, por lo tanto, en el $82 \%$ restante de las muestras se presentó la forma clásica. En esta misma línea Chaney et al. (1996) reportaron que la forma clásica se presentó en el $64 \%$ de su muestra, cifra inversa a lo señalado por Lucien (1909) quien en una muestra de 51 pies describió la presencia de fascículos adicionales en un $73 \%$.

Dentro de las variantes menos comunes vale la pena destacar la expuesta por Chaney et al. quienes informaron además la existencia de una cabeza y tendón variable hacia el quinto dedo, presente en $18 \%$ de la muestra, lo que concuerda con lo expuesto por Cruveilheir (1843) quien mencionó que no es raro poder observarlo. Aún menos frecuente es la variante presentada por Hallisy (1930) quien señaló que el ECD recibió proyecciones del músculo flexor largo de los dedos.
La historia de la cirugía reconstructiva se ha basado en buscar cada vez mejores estrategias de abordaje para poder brindar así mejores resultados a los defectos tisulares complejos. Lo anterior se ha sustentado tanto en fuertes fundamentos anatómicos, como también en el uso de nuevas tecnologías, en la estandarización de las técnicas y el manejo preoperatorio. Esto no sólo busca optimizar resultados funcionales y estéticos, sino que disminuye las secuelas del sitio donante. Al respecto, Chang et al. (1986) indicaron varios argumentos que justifican el uso del músculo ECD como un muy buen colgajo; señalando en primer lugar que la arteria dorsal del pie y las venas safenas magna y parva que vascularizan esta región son más grandes y fáciles de disecar y anastomosar. Así mismo, la capa subcutánea de tejido adiposo del colgajo dorsal del pie es más delgada y la piel más fina, con mayor elasticidad y fácil de moldear. Además, se puede transferir junto con ramos del nervio fibular profundo (NFP) en el mismo tiempo operatorio, lo que permite una restauración más temprana de la inervación.

En este contexto, Thompson (1971) fue uno de los primeros cirujanos en usar el músculo ECD como colgajo libre en el tratamiento de la parálisis facial unilateral y con posterioridad Thompson \& Gustavson (1976) lo ocuparon como injerto en parálisis faciales bilaterales. En esa misma línea Huang et al. (2009) y Oh \& Chung (2011) describieron el desarrollo de estas técnicas informando diversos grados de restauración del movimiento voluntario. En otros ámbitos quirúrgicos, Westlin et al. (2003) y Hirschmann et al. (2008) utilizaron este músculo para la corrección de la inestabilidad lateral de tobillo. Por su parte Scott \& Tuten (2007) y Van Renterghem \& De Ridder (2011) utilizaron el colgajo del músculo ECD como tejido de interposición en barras calcáneo naviculares y a su vez Landi et al. (1985), Hing et al. (1987), Giordano et al. (1989) y Pai et al. (2000) lo hicieron para la corrección de defectos en tejidos blandos de la región distal de la pierna y del pie.

A pesar de que en las experiencias clínicas se reportaron valores referentes a las áreas de extensión de estos músculos, los estudios que incluyen valores biométricos al respecto son escasos y la mayor parte de los estudios clínicos se enfocaron principalmente en las técnicas de utilización de los músculos ECD y ECH en objetivos quirúrgicos. Al respecto Sirasanagandla et al. analizaron 44 miembros inferiores estimando una longitud, ancho y área de extensión promedio de la suma de los músculos ECD y ECH para el lado derecho de $7,39 \pm 0,71 \mathrm{~cm} ; 4,10 \pm 0,37 \mathrm{~cm}$ y $30,5 \pm$ $4,78 \mathrm{~cm}^{2}$ y de $7,20 \pm 0,84 \mathrm{~cm} ; 3,90 \pm 0,37 \mathrm{~cm}$ y $28,4 \pm 5,35$ $\mathrm{cm} 2$ para el lado izquierdo, respectivamente. Estos valores se acercan al promedio del área de extensión presentado por Landi et al. de $33,50 \mathrm{~cm}^{2}$; a los de Hing et al. de $27 \mathrm{~cm}^{2}$ y a los de Pai et al. de $30 \mathrm{~cm}^{2}$, sin embargo, son considerable- 
CAAMAÑO-BINIMELIS, C.; RIVEROS, A.; SOUSA-RODRIGUES, C. \& OLAVE, E. Biometría de los músculos extensor corto de los dedos y extensor corto del hallux en individuos brasileños: análisis anatómico para su uso como colgajo muscular en cirugías reconstructivas. Int. J. Morphol., 38(4):1010-1017, 2020.

mente superiores con las informadas por Giordano et al. de $20,20 \mathrm{~cm}^{2}$ y del Piñal \& Herrero (2000) con un promedio de $19,71 \mathrm{~cm}^{2}$ al lado derecho y $19,73 \mathrm{~cm}^{2}$ al lado izquierdo.

En lo que respecta al dato biométrico de longitud, Casal et al. (2010) se centró en estudiar las características del aparato extensor del hallux para lo cual estudió los músculos ELH y ECH, obteniendo una longitud total promedio para este último de 10,30 $\pm 0,80 \mathrm{~cm}$ al lado derecho y 10,90 $\pm 0,20$ $\mathrm{cm}$ al lado izquierdo en hombres y de $10,10 \pm 0,70 \mathrm{~cm}$ al lado derecho y 10,00 \pm 0,10 al lado izquierdo en mujeres.

Por otra parte, la inervación de los músculos ECD y ECH está dado por el NFP el cual se dispone profundo al músculo ELD, anterior a la membrana interósea, y acompañado de la arteria tibial anterior hasta el tobillo donde se divide en un ramo terminal medial y lateral (Sarrafian \& Kelikian). En el mismo sentido Standring describió que el ramo terminal lateral del NFP cruza el tobillo de forma oblicua para posteriormente presentar una expansión similar a un pseudoganglio y así finalmente inervar a los músculos ECD y ECH.

Para Horwitz (1938) esta división se realizó en la mayoría de los casos (98 \%) a 1,3 cm proximal a la articulación del tobillo y en el menor de los casos $(2 \%)$ esta ramificación se presentó a nivel de la articulación del tobillo o por sobre $6,4 \mathrm{~cm}$ proximal a esta articulación. Los datos respecto del diámetro de los nervios implicados en el control de los músculos ECD y ECH son escasos, en este sentido Sarrafian \& Kelikian informaron que estos diámetros variaron de 1 a $3 \mathrm{~mm}$.

Basado en lo anterior, el propósito del presente estudio fue establecer la longitud, ancho y área de extensión de estos músculos, acompañado de la determinación de las distancias desde estos músculos respecto a la cuña medial y la base del quinto metatarsiano. Así mismo, se describieron las variantes musculares encontradas y se determinó los puntos motores de inervación de estos dos músculos. Estos hallazgos se hacen relevantes por su aporte anatomo-quirúrgico respecto a la caracterización biométrica de los músculos de la región dorsal del pie.

\section{MATERIAL Y MÉTODO}

Se utilizaron 36 miembros inferiores (18 pies derechos y 18 izquierdos) de individuos adultos, brasileños, pertenecientes al laboratorio de Anatomía de la Universidad Federal de Alagoas (UFAL), Maceió, Brasil. De esta muestra, el $94 \%$ (34 casos) correspondió a pies de cadáveres masculinos. Dichos cadáveres se encontraban fijados bajo inmersión en una solución con formaldehído al $10 \%$. Esta investigación se realizó utilizando una metodología de carácter cuantitativo, no experimental, transeccional y descriptiva, para lo cual se realizó una disección del compartimento anterior de la pierna y de la región dorsal del pie. Esta disección se realizó utilizando una técnica topográfica por planos, utilizando para ello material quirúrgico ad-hoc con los cadáveres en posición decúbito dorsal.

Luego de remover la piel y el tejido subcutáneo de la región anterior de la pierna y dorsal del pie se escindió el músculo ELD y de traccionó desde su origen hasta el punto en que sus tendones se proyectaron sobre la falange proximal de los cuatro dedos mediales. A continuación, se realizó el mismo procedimiento con el músculo ELH el cual al ser removido dejó expuesto tanto los vientres y tendones de los músculos ECD y del ECH como también al NFP acompañado de la arteria y venas tibiales anteriores. El nervio fue separado desde el paquete neurovascular del compartimento anterior de la pierna y región dorsal del tobillo mediante técnicas de divulsión, siguiendo la división de sus ramos terminales hasta el punto en que el ramo lateral de este nervio emitió los ramos para los músculos ECD y ECH.

Posterior a esta disección, se midió la longitud de los músculos ECH y ECD y estableciendo como longitud para este último el promedio de sus cabezas componentes. Posteriormente se midió el ancho y área de extensión considerando ambos músculos. Con la finalidad de colaborar en los procedimientos quirúrgicos en el abordaje de la obtención del colgajo de la región dorsal del pie, se midió la distancia existente entre el margen lateral del músculo ECD a la base del $5^{\circ}$ metatarsiano y desde la cuña medial al margen medial del músculo ECH, estableciendo previamente como ancho del pie, la distancia que comunica ambos reparos óseos.

Por último, se determinó si la división del NFP se produjo proximal, al mismo nivel o distal de la línea intermaleolar (LIM). A continuación, se midió el diámetro del NFP, la longitud y el diámetro de cada uno de sus ramos terminales. Luego y previa división de los músculos ECD y $\mathrm{ECH}$ en tercios, se establecieron los puntos motores (Pm) de cada uno de estos músculos.

Todas las mediciones fueron realizadas con un cáliper digital Mitutoyo ${ }^{\circledR}$ de $0,01 \mathrm{~mm}$ de precisión y complementariamente las muestras disecadas fueron fotografiadas con una cámara réflex digital Nikon ® D5300. Los resultados se ingresaron a una base de datos Microsoft Excel (B) y se calcularon las medidas de tendencia central de cada una de las variables registradas. 


\section{RESULTADOS}

El músculo ECD junto al músculo ECH estuvieron presentes en todos los miembros inferiores analizados, mencionando que en todos los casos el vientre muscular del $\mathrm{ECH}$ se encontró fusionado con el vientre del músculo ECD desde su origen. La presentación más frecuente del músculo ECD fue en su forma clásica en un $83 \%$ de los casos, obteniendo un $17 \%$ de muestras con presencia de variantes relacionadas con la existencia de tendones accesorios. De éstas, un $83 \%$ correspondió a muestras que presentaron un tendón accesorio (Fig. 1). En $17 \%$ $\%$ de los casos se presentaron dos tendones anexos observados a partir de una cabeza accesoria (Fig. 2). Todos los tendones supernumerarios se observaron entre la primera y segunda cabeza. Del total de pies que presentaron variantes anatómicas, $67 \%$ fue en el lado derecho.

Respecto a la biometría del músculo ECD, presentó una longitud promedio, considerado desde su origen hasta el término de cada una de sus cabezas de $6,89 \pm 1,64 \mathrm{~cm}$ en las del lado derecho y de $6,91 \pm 1,64 \mathrm{~cm}$ en las muestras del lado izquierdo, predominando la longitud de su tercera cabeza, con $7,89 \pm 1,70 \mathrm{~cm}$ en el lado derecho y 7,98 \pm $1,32 \mathrm{~cm}$ en el izquierdo, obteniendo como valores máximos 10,88 y $10,41 \mathrm{~cm}$ y como valores mínimos 3,34 y $6,10 \mathrm{~cm}$ en los pies derechos e izquierdos, respectivamente.

El ancho medido en el punto medio de su longitud alcanzó un valor promedio de $3,81 \pm 0,42 \mathrm{~cm}$ con un intervalo entre 4,49 y $3,05 \mathrm{~cm}$, lado derecho y de $3,68 \pm 0,46 \mathrm{~cm}$ con intervalo entre 4,90 y $2,98 \mathrm{~cm}$, al lado izquierdo. El área de extensión promedio del músculo ECD obtenido al lado derecho fue de $31,98 \pm 7,60 \mathrm{~cm}^{2}$ con un intervalo entre 48,80 y $20,79 \mathrm{~cm}^{2}$ y al lado izquierdo de 30,75 $\pm 7,61 \mathrm{~cm}^{2}$ con un intervalo entre $51,01 \mathrm{y}$ $20,39 \mathrm{~cm}^{2}$. Los hallazgos de promedio de las longitudes de cada vientre de los músculos ECD y ECH se encuentran resumidas en la Tabla I. Los promedios de longitud, ancho y área de extensión del total de las muestras se exponen en la Tabla II.
$\mathrm{Al}$ analizar los valores exclusivos para el músculo ECH se obtuvo una longitud total promedio (vientre más su tendón) de $11,47 \pm 1,31 \mathrm{~cm}$ con intervalos entre 13,12 y $8,93 \mathrm{~cm}$ en los pies del lado derecho y $11,83 \pm$ $1,19 \mathrm{~cm}$ con intervalos entre 13,80 y $8,93 \mathrm{~cm}$ en los pies del lado izquierdo.

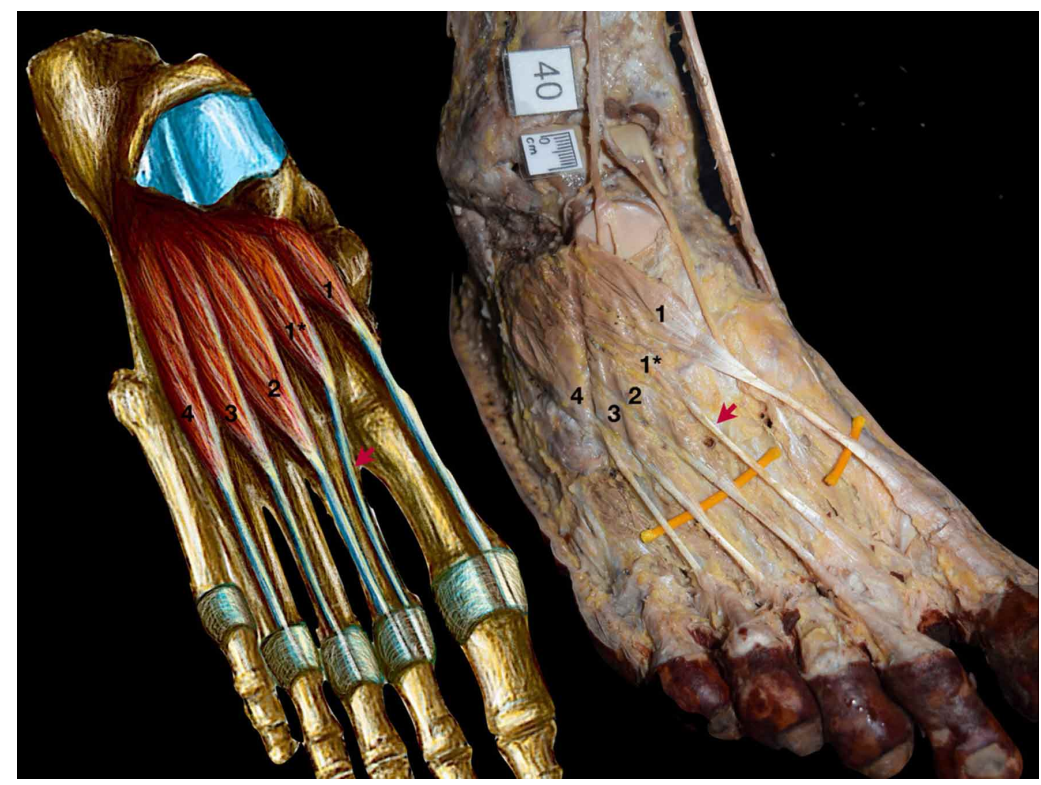

Fig. 1. 1. Músculo extensor corto del hallux (primera cabeza del extensor corto de los dedos), $1^{*}$. Cabeza accesoria del músculo extensor corto de los dedos 2 . Segunda cabeza del músculo extensor corto de los dedos, 3 . Tercera cabeza del músculo extensor corto de los dedos, 4. Cuarta cabeza del músculo extensor corto de los dedos, Flecha roja: tendón accesorio.

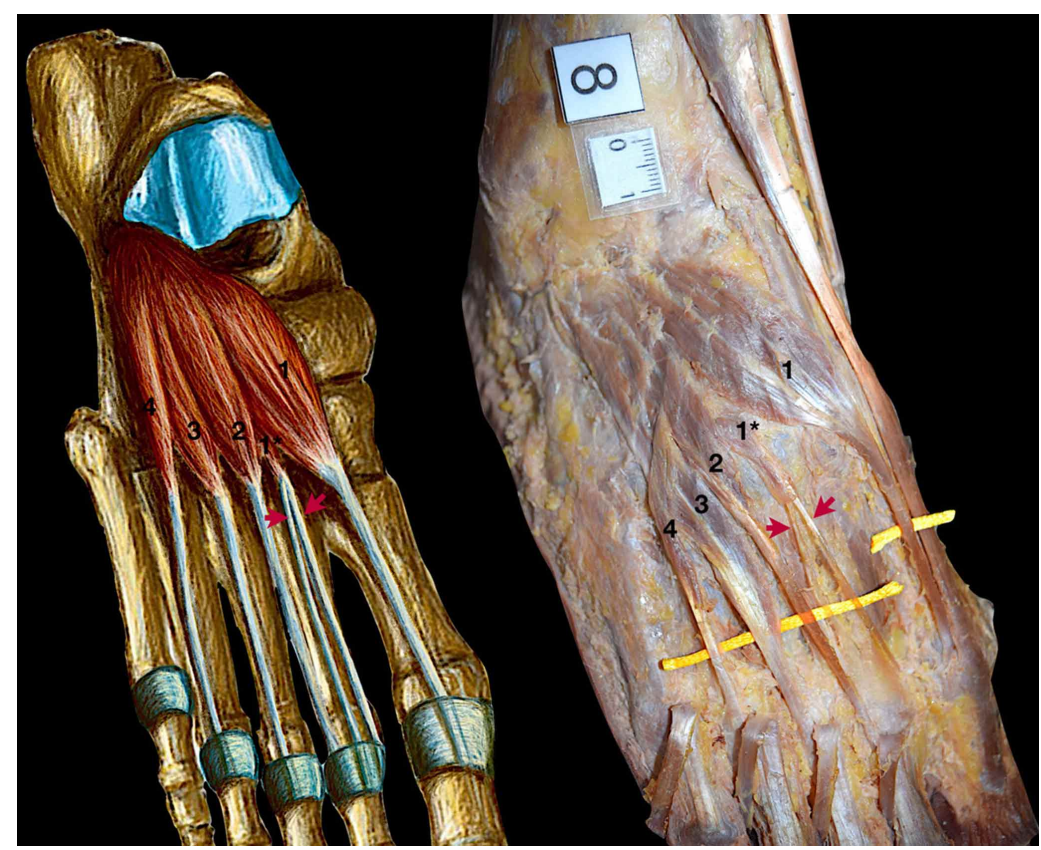

Fig. 2. 1. Extensor corto del hallux (primera cabeza del extensor corto de los dedos), $1 *$. Cabeza accesoria del extensor corto de los dedos 2. Segunda cabeza del extensor corto de los dedos, 3 . Tercera cabeza del extensor corto de los dedos, 4. Cuarta cabeza del extensor corto de los dedos, Flechas rojas: tendones accesorios. 
CAAMAÑO-BINIMELIS, C.; RIVEROS, A.; SOUSA-RODRIGUES, C. \& OLAVE, E. Biometría de los músculos extensor corto de los dedos y extensor corto del hallux en individuos brasileños: análisis anatómico para su uso como colgajo muscular en cirugías reconstructivas. Int. J. Morphol., 38(4):1010-1017, 2020.

Tabla I. Longitud de las cabezas de los músculos extensor corto de los dedos y extensor corto del hallux.

\begin{tabular}{|c|c|c|c|c|c|c|c|c|}
\hline \multirow[b]{2}{*}{ Cabezas } & \multicolumn{3}{|c|}{ Lado derecho } & \multirow[b]{2}{*}{$\begin{array}{l}\text { Desviación } \\
\text { estandar }\end{array}$} & \multirow[b]{2}{*}{$\begin{array}{l}\text { Mín } \\
(\mathrm{cm})\end{array}$} & \multicolumn{3}{|c|}{ Lado izquierdo } \\
\hline & $\begin{array}{l}\text { Mín } \\
(\mathrm{cm})\end{array}$ & $\begin{array}{l}\text { Máx } \\
(\mathrm{cm})\end{array}$ & $\begin{array}{c}\text { Promedio } \\
\text { Longitud }(\mathrm{cm})\end{array}$ & & & $\begin{array}{l}\text { Máx } \\
(\mathrm{cm})\end{array}$ & $\begin{array}{l}\text { Promedio Longitud } \\
(\mathrm{cm})\end{array}$ & $\begin{array}{l}\text { Desviación } \\
\text { estandar }\end{array}$ \\
\hline 1er ${ }^{\mathrm{a}}$ C. $(\mathrm{ECH})$ & 3,89 & 6,38 & 5,14 & 0,76 & 3,64 & 6,98 & 5,30 & 0,90 \\
\hline $2^{\mathrm{a}}$ C. (ECD) & 5,10 & 9,73 & 7,25 & 1,17 & 5,82 & 10,19 & 7,56 & 1,17 \\
\hline $3^{\text {era }}$ C. (ECD) & 3,34 & 10,88 & 7,89 & 1,70 & 6,10 & 10,41 & 7,98 & 1,32 \\
\hline $4^{\mathrm{a}}$ C. (ECD) & 4,81 & 9,07 & 7,27 & 1,36 & 3,31 & 9,52 & 6,80 & 1,68 \\
\hline
\end{tabular}

Tabla II. Biometría de los músculos extensor corto de los dedos y extensor corto del hallux.

\begin{tabular}{|c|c|c|c|c|c|c|c|c|}
\hline \multicolumn{4}{|c|}{ Lado derecho } & & \multicolumn{4}{|c|}{ Lado izquierdo } \\
\hline Medidas & Mín & Máx & Promedio & $\begin{array}{c}\text { Desviación } \\
\text { estandar }\end{array}$ & Mín & Máx & Promedio & $\begin{array}{c}\text { Desviación } \\
\text { estandar }\end{array}$ \\
\hline Longitud (cm) & 5,37 & 8,61 & 6,89 & 1,64 & 5,24 & 8,79 & 6,91 & 1,64 \\
\hline Ancho (cm) & 3,05 & 4,49 & 3,81 & 0,42 & 2,98 & 4,90 & 3,68 & 0,46 \\
\hline Área de extensión $\left(\mathrm{cm}^{2}\right)$ & 20,79 & 48,80 & 31,98 & 7,60 & 20,39 & 51,01 & 30,75 & 7,61 \\
\hline
\end{tabular}

Las mediciones relacionadas con la distancia entre el margen lateral del músculo ECD a la base del $5^{\circ}$ metatarsiano fue en promedio de 1,72 $\pm 0,41 \mathrm{~cm}$ con un intervalo de 2,40 y $0,41 \mathrm{~cm}$ en los pies derechos y de $1,87 \pm 0,36 \mathrm{~cm}$ con un intervalo entre 2,63 y $1,31 \mathrm{~cm}$ en los pies izquierdos. La distancia comprendida entre la cuña medial al margen medial del músculo ECH fue en promedio de $1,97 \pm 0,43 \mathrm{~cm}$ con un valor máximo de 2,66 y un valor mínimo de 1,29 al lado derecho y de 2,01 cm $\pm 0,62 \mathrm{~cm}$ con un intervalo de 3,62 y 1,05 cm., al lado izquierdo. La biometría de estas distancias respecto a la posición los músculos ECD y ECH se encuentran expuestos en la Tabla III.

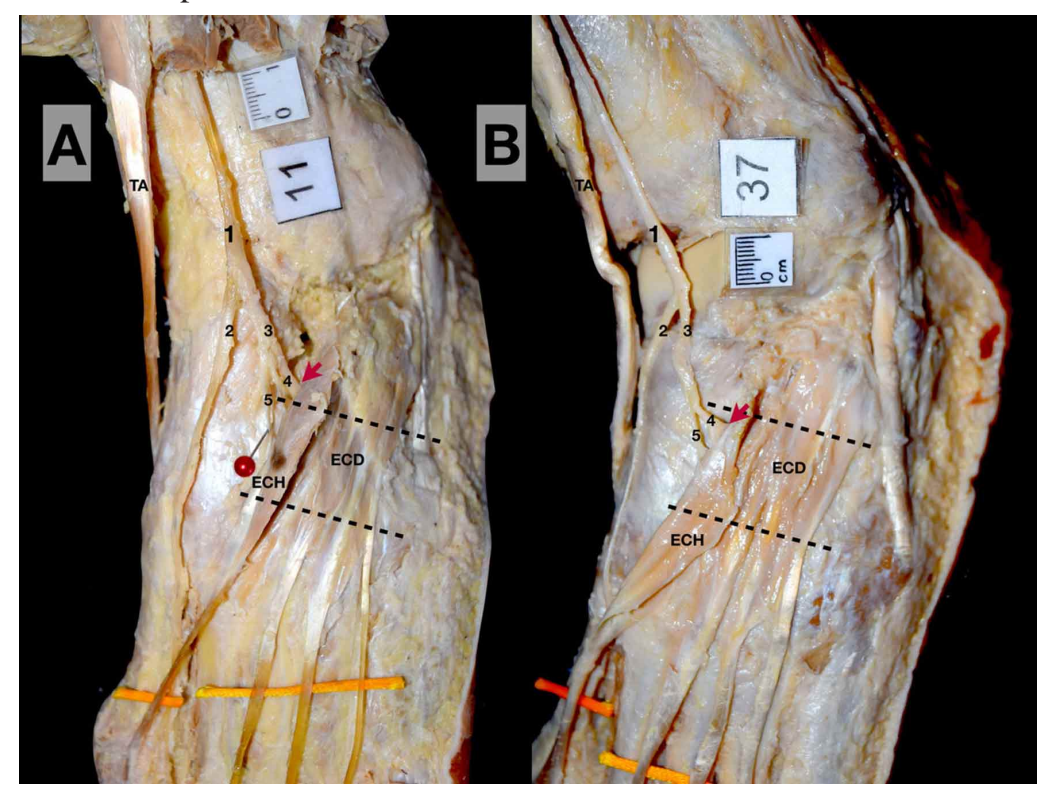

Fig. 3. Cara dorsal pies izquierdos. Flecha roja indica punto motor del ramo del nervio fibular profundo para el músculo extensor corto de los dedos, A. En el tercio proximal, B. En el tercio medio, 1. Nervio fibular profundo, 2. Ramo medial del nervio fibular profundo, 3. Ramo lateral del nervio fibular profundo, 4. Ramo para el músculo extensor corto de los dedos, 5. Ramo para el músculo extensor corto del hallux, ECD: músculo extensor corto de los dedos, ECH: músculo extensor corto del hallux, TA: músculo tibial anterior.
Respecto a la división de los ramos terminales del NFP, en un $3 \%$ de los casos se observó a más de $6,4 \mathrm{~cm}$ proximal a la LIM, en un $39 \%$ esta división se efectuó a una distancia de $1,3 \mathrm{~cm}$ proximal a la LIM, en un $27 \%$ se realizó a nivel de la LIM y en un $30 \%$ se dividió distal a ella. El diámetro del NFP fue medido en todos los casos a $10 \mathrm{~cm}$ proximal a la LIM y alcanzó un promedio de 2,05 \pm 0,48 $\mathrm{mm}$ con un intervalo entre 2,77 y $1,23 \mathrm{~mm}$, en los pies del lado derecho y de 2,14 $\pm 0,41 \mathrm{~mm}$ con un intervalo entre 2,78 y $1,37 \mathrm{~mm}$, en los pies del lado izquierdo.

Finalmente, los puntos motores en el músculo ECD se distribuyeron en $64 \%$ de los casos en el tercio proximal de este músculo y en un $36 \%$ en el tercio medio. Al comparar estas distribuciones por lateralidad se observó que en el lado derecho, en un $72 \%$ de los casos los Pm se localizaron en la cara profunda de su tercio proximal, dejando al $28 \%$ restante en su tercio medio. Por su parte al lado izquierdo, los Pm se ubicaron en un $56 \%$ en el tercio proximal, en un $44 \%$ en el tercio medio (Fig. 3). En cuanto a la distribución de los Pm sobre el músculo ECH, éstos se distribuyeron en un $28 \%$ en el tercio proximal, en un $64 \%$ en el tercio medio y en un $8 \%$ en el tercio distal. Al revisar la distribución por lateralidad se encontró que en el lado derecho fue de $44 \%$ en el tercio proximal, $50 \%$ en el tercio medio y $6 \%$ en el tercio distal, mientras que en el lado izquierdo fue de $11 \%$ en el tercio proximal, 78 $\%$ en el tercio medio y $11 \%$ en el tercio distal. 
CAAMAÑO-BINIMELIS, C.; RIVEROS, A.; SOUSA-RODRIGUES, C. \& OLAVE, E. Biometría de los músculos extensor corto de los dedos y extensor corto del hallux en individuos brasileños: análisis anatómico para su uso como colgajo muscular en cirugías reconstructivas. Int. J. Morphol., 38(4):1010-1017, 2020.

Tabla III. Biometría de la distancia desde los márgenes lateral y medial del pie respecto a la posición de los músculos ECD y ECH.

\begin{tabular}{lcccccc}
\hline Medidas & $\begin{array}{c}\text { Ancho del } \\
\text { pie } \\
(\mathrm{cm})\end{array}$ & $\begin{array}{c}\text { Lado derecho } \\
\text { Distancia desde } \\
\text { cuña medial }-\end{array}$ & $\begin{array}{c}\text { Distancia } 5^{\circ} \text { Mtt - } \\
\text { ECD }(\mathrm{cm})\end{array}$ & $\begin{array}{c}\text { Ancho del pie } \\
(\mathrm{cm})\end{array}$ & $\begin{array}{c}\text { Lado izquierdo } \\
\text { Distancia desde } \\
\text { Cuña medial - } \\
\text { ECH }\end{array}$ & $\begin{array}{c}\text { Distancia 5 } \\
\text { ECD } \\
(\mathrm{cm})\end{array}$ \\
\hline Promedio & 6,63 & 1,97 & 1,72 & 6,71 & 2,01 & 1,87 \\
Mínimo & 5,61 & 1,29 & 0,81 & 5,64 & 1,05 & 1,31 \\
Máximo & 8,25 & 2,66 & 2,40 & 7,94 & 3,62 & 2,63 \\
D. Standard & 0,76 & 0,43 & 0,41 & 0,71 & 0,62 & 0,36 \\
\hline
\end{tabular}

\section{DISCUSIÓN}

El músculo ECD se presentó en su forma clásica en un $83 \%$ de nuestra serie, y tan sólo en un $17 \%$ presentó de variantes anatómicas. Lo anterior, concuerda con lo señalado por Sirasanagandla et al. quienes reportaron que un $82 \%$ de los casos presentó dicha forma, dato similar al de Chaney et al., quienes informaron un $64 \%$. Todos estos datos son superiores a los señalado por Lucien, quien obtuvo un $27 \%$ de pies con la formación clásica. En relación con las variantes informadas, Macalister; Testut \& Latarjet (1972) y Sarrafian \& Kelikian describieron la presencia de tendones accesorios emitidos por la segunda y tercera cabeza del músculo ECD. Al respecto, nuestro estudio ha mostrado que un $14 \%$ de los casos presentaba un (1) tendón accesorio, cifra que duplica lo informado por Sirasanagandla et al., sin embargo, la variación de dos tendones accesorios presente en el $3 \%$ de la presente muestra, concuerda con el $2 \%$ informado por estos mismos autores. Así mismo, ambas cifras son similares al $4 \%$ reportado por Chaney et al. Al respecto, Lucien y Papalmitiades (1948), señalaron que la presencia de tendones supernumerarios se sustenta en dos argumentos: el primero de ellos, basado en estudios de anatomía comparada, describieron la existencia de una migración de este músculo desde la región fibular a la región tarso-metatarsiana; el segundo, complementado por Sirasanagandla et al., estableció que, bajo observaciones de embriones humanos de $30 \mathrm{~mm}$, se pudieron reconocer dos masas celulares independientes que se encargaron de la formación de los músculos ECD y ECH. En estas masas, que se fusionan para generar un vientre muscular único, algunas células pueden originar la aparición de cabezas supernumerarias o fascículos accesorios.

En cuanto a la biometría del músculo ECD, si bien nuestra investigación arrojó una longitud promedio del total de $6,90 \mathrm{~cm}$, la cual se asemeja a los registros presentados por Sirasanagandla et al. $(7,30 \mathrm{~cm})$, existió con este autor, una diferencia en la metodología de la medición, ya que obtuvimos la longitud total del músculo, a partir del promedio de longitud de todas las cabezas y no por la distancia medida desde el calcáneo al segundo dedo, como este autor in- formó. A pesar de ello, ambas investigaciones muestran valores superiores a lo informado por Giordano et al., los que presentaron un valor promedio de $5,10 \mathrm{~cm}$ en una muestra de 20 pies, en donde predominó el sexo femenino. La misma diferencia se presentó con el estudio de del Piñal \& Herrero, quienes obtuvieron una longitud de $5,11 \mathrm{~cm}$, obtenida a través del promedio entre la longitud máxima y mínima de sus cabezas.

Al medir de forma independiente la longitud promedio del músculo ECH incluyendo su tendón, nuestros registros $(11,65 \mathrm{~cm})$ concuerdan con Casal et al., quienes obtuvieron $10,50 \mathrm{~cm}$. Estos datos reafirman el hecho de que este grupo muscular es un candidato óptimo para la realización de cirugías que requieran colgajos musculares.

Al comparar el ancho promedio con autores como del Piñal \& Herrero $(3,78 \mathrm{~cm})$ y Sirasanagandla et al. (4,0 $\mathrm{cm}$ ) los valores fueron similares, ya que obtuvimos $3,75 \mathrm{~cm}$, a pesar que el ancho se registró en el punto medio de la longitud de los músculos y del Piñal \& Herrero lo hicieron midiendo el ancho en el punto de origen de estos músculos y Sirasanagandla et al., en el punto medio existente entre el origen de dichos músculos hasta el punto proximal en donde se independizó el tendón destinado al segundo dedo.

Respecto al área promedio de extensión del músculo ECD y ECH, Giordano et al. y del Piñal \& Herrero han señalado valores de $20,20 \mathrm{~cm}^{2}$ y $19,72 \mathrm{~cm}^{2}$, respectivamente. Lo anterior es notoriamente inferior a los valores indicados por Hing et al. de $27 \mathrm{~cm}^{2}$ y éstos, respecto a los valores expuestos por Landi et al. de $33,50 \mathrm{~cm}^{2}$, Pai et al. de $30 \mathrm{~cm}^{2}$ y Sirasanagandla et al. de $31,24 \mathrm{~cm}^{2}$. Nuestro valor promedio de $31,37 \mathrm{~cm}^{2}$ se acerca a estos últimos estudios. Dichas diferencias son consistentes al considerar el ancho y el área informadas por cada estudio.

La inervación del músculo ECD y ECH ha sido descrita en la literatura anatómica tradicional; textos como Testut \& Latarjet; Rouvière \& Delmas (2005) y Moore \& Dalley 
CAAMAÑO-BINIMELIS, C.; RIVEROS, A.; SOUSA-RODRIGUES, C. \& OLAVE, E. Biometría de los músculos extensor corto de los dedos y extensor corto del hallux en individuos brasileños: análisis anatómico para su uso como colgajo muscular en cirugías reconstructivas. Int. J. Morphol., 38(4):1010-1017, 2020.

(2006) han señalado que esta inervación es otorgada por el ramo lateral del NFP, sin embargo, otros autores han descrito la presencia de un ramo proveniente del nervio fibular superficial (NFS) denominado nervio fibular profundo accesorio, el cual le otorga inervación a ambos músculos (Tzika et al., 2012; Tomaszewski et al., 2016). Esta variante nerviosa no fue evidenciada en nuestras muestras analizadas. Lo anterior refuerza la condición de donante de este músculo en cirugías que requieran el uso de colgajos, ya que la probabilidad de que existan variantes nerviosas en este grupo muscular es baja.

En lo que respecta a la división del NFP en ramos terminales lateral y medial, Horwitz sostuvo que en el 98 $\%$ de su muestra, esta división se produjo a $1,3 \mathrm{~cm}$ proximal a la línea articular del tobillo, mientras que en nuestros registros dicha división en este punto se registró sólo en un $39 \%$ de los casos. Así mismo, la división en un rango superior a $6,4 \mathrm{~cm}$ proximal a esta línea o a nivel de ella informada por este autor en un $2 \%$, es alejada a lo obtenido en nuestra investigación, en donde la división en un rango superior a $6,4 \mathrm{~cm}$ proximal a la línea articular estuvo presente en un $3 \%$ de los casos, a nivel de ella en un 27 $\%$ y en un punto distal respecto a esta referencia en un 30 $\%$.

Cabe destacar que si bien hay investigaciones que describen la localización de los Pm en diferentes músculos, (Olave et al., 2002; Olave et al., 2012; Riveros et al., 2016), no se encontraron estudios cadavéricos que expongan el registro de Pm en el músculo ECD. Lo anterior realza el valor de esta investigación como un interesante aporte a la biometría muscular, contribuyendo al conocimiento de la inervación de este músculo, y a la selección que se estime del mismo en futuras intervenciones médico-quirúrgicas.

Finalmente cabe destacar que este estudio propone la distancia más frecuente que presenta el origen de los márgenes lateral y medial de este músculo respecto a los reparos óseos estimados para medir el ancho del pie y por lo tanto entrega una valiosa información respecto a los sitios topográficos seguros para la realización de abordajes quirúrgicos en el dorso del pie.

CAAMAÑO-BINIMELIS, C.; RIVEROS, A.; SOUSARODRIGUES, C. \& OLAVE, E. Biometry of the extensor digitorum brevis and extensor hallucis brevis muscles in brazilian individuals: anatomical analysis for use as a muscle flap in reconstructive surgeries. Int. J. Morphol., 38(4):1010-1017, 2020.

SUMMARY: The extensor digitorum brevis muscle (EDB) is located along with the extensor hallucis brevis (EHD) in the dorsal region of the foot and are responsible for collaborating with the agonist action of the extensor digitorum longus muscles (EDL) ) and extensor hallucis longus (EHL) in the extension of the four medial fingers in the metatarsophalangeal joints. This complementary condition allows its surgical transfer to other receptor regions without affecting the extensor functionality of the toes, which is why during the last decades it has become an important tissue for the reconstruction of tissue injuries, however, there are few studies biometrics that describe it in detail. The objective of this investigation was to establish the length, width and area of extension of these muscles accompanied by the determination of their distances from the medial wedge and the base of the fifth metatarsal for surgical use. In addition to the above and after the description of the muscle variants found, the innervation motor points of these two muscles were determined. To do this, 36 formalized lower limbs of Brazilian adult individuals belonging to the Federal University of Alagoas (UFAL), Maceió, Brazil, were studied. Measurements were made with a $0.01 \mathrm{~mm}$ precision Mitutoyo caliper obtaining a length, width and area of $6.89 \pm 1.64 \mathrm{~cm}, 3.81 \pm 0.42 \mathrm{~cm}$ and $31.98 \pm 7.60 \mathrm{~cm}^{2}$ on the right side of $6.91 \pm 1.64 \mathrm{~cm}, 3.68 \pm 0.46 \mathrm{~cm}$ and $30.75 \pm 7.61 \mathrm{~cm}^{2}$ on the left, respectively. In $17 \%$ of cases there is presence of accessory tendons for the EDB muscle. The distance from the medial margin of the EHB muscle with respect to the medial wedge and the lateral margin of the EDB muscle to the base of the fifth metatarsal was $1.97 \pm 0.43$ and $1.72 \pm 0.41$ on the right side and 2,01 \pm 0.62 and $1.87 \pm 0.36$ on the left side respectively. Motor points $(\mathrm{Pm})$ predominated in $64 \%$ in the middle third of the EHB muscle and in $64 \%$ in the proximal third of the EDB muscle. These results are a significant contribution both for those who perform orthopedic surgery and for detailed knowledge of the dorsal foot anatomy.

KEY WORDS: Anatomy; Foot dorsum; Innervation; extensor digitorum brevis muscle; extensor hallucis brevis muscle; motor points; biometry.

\section{REFERENCIAS BIBIOGRÁFICAS}

Bergman, R.; Thompson, S. \& Afifi, A. Compendium of Human Anatomic Variation. Baltimore, Urban \& Schwarzenberg, 1988.

Casal, D.; Pais, D.; Almeida, M. A.; Bilhim, T.; Santos, A. \& Goyri-O’Neill, J. Morphometric analysis of the extensor tendons of the hallux and potential implications for tendon grafting. Eur. J. Anat., 14(1):11-8, 2010.

Chaney, D. M.; Lee, M. S.; Khan, M. A.; Krueger, W. A.; Mandracchia, V. J. \& Yoho, R. M. Study of ten anatomical variants of the foot and ankle. J. Am. Podiatr. Med. Assoc., 86(11):532-7, 1996.

Chang, T.; Zhu, S. \& Wang, Z. Microsurgical Principles, Techniques and Applications. Singapore, World Scientific Publishing, 1986.

Chattar-Cora, D. \& Pederson, W. C. Experience with the extensor digitorum brevis muscle flap for foot and ankle reconstruction. Ann. Plas. Surg., 57(3):289-94, 2006

Cruveilheir, J. Traitè d'Anatomie Descriptive. 2a éd. Paris, B.chet Jeune, 1843.

del Piñal, F. \& Herrero, F. Extensor digitorum brevis free flap: anatomic study and further clinical applications. Plast. Reconstr. Surg., 105(4):1347-56, 2000. 
Giordano, P. A.; Argenson, C. \& Pequignot, J. P. Extensor digitorum brevis as an island flap in the reconstruction of soft-tissue defects in the lower limb. Plast. Reconstr. Surg., 83(1):100-9, 1989.

Hallisy, J. E. The muscular variations in the human foot. A quantitative study. General results of the study: I. Muscles of the inner border of the foot and the dorsum of the great toe. Am. J. Anat., 45(3):411-42, 1930

Hing, D. N.; Buncke, H. J. \& Alpert, B. S. Applications of the extensor digitorum brevis muscle for soft tissue coverage. Ann. Plast. Surg., 19(6):530-7, 1987.

Hirschmann, M. T.; Friederich, N. \& Müller, C. Anatomic reconstruction of a chronically unstable calcaneocuboid joint with augmented extensor digitorum brevis muscle flap: a new method. Foot Ankle Int., 29(12):1258-61, 2008.

Horwitz, T. Normal anatomy and variations of the peripheral nerves of the leg and foot. Application in operations for vascular diseases: study of one hundred specimens. Arch. Surg., 36(4):626-36, 1938.

Huang, W. Q,; Fang, B. R; Fang, X. Q. \& Wang C. Extensor digitorum brevis and extensor hallusis brevis transplantation for treatment of longstanding facial paralysis. Chin. J. Traumatol., 12(1):3-9, 2009.

Landi, A.; Soragni, O. \& Monteleone, M. The extensor digitorum brevis muscle island flap for soft-tissue loss around the ankle. Plast. Reconstr. Surg., 75(6):892-7, 1985.

Lucien, M. Les chefs accessoires du muscle court extenseur des orteils chez l'homme. Bibl. Anat. (Basel), 14:148, 1909.

Macalister, A. Additional observations on muscular anomalies in human anatomy. (Third series) With a catalogue of the principal muscular variations hitherto published. Trans. R. Ir. Acad. Sci., 25:1-134, 1875.

McCraw, J. B. \& Furlow, L. T. Jr. The dorsalis pedis arterialized flap. A clinical study. Plast. Reconstr. Surg., 55(2):177-85, 1975.

Moore, K. \& Dalley, A. Anatomía con Orientación Clínica. $7^{\mathrm{a}}$ ed. Buenos Aires, Médica Panamericana, 2006.

Oh, S. J. \& Chung, C. H. Upper-lip reconstruction using a free dorsalis pedis flap incorporating the extensor hallucis and digitorum brevis muscles. J. Craniofac. Surg., 22(3):998-9, 2011.

Olave, E.; Gabrielli, C.; Braga, M. T. T. \& del Sol, M. Biometric aspects of the motor branches of the musculocutaneous nerve to the brachial muscle. Rev. Chil. Anat., 20(2):231-6, 2002.

Olave, E.; Retamal, P.; Galaz, C. \& Cruzat, C. Innervation and localization of the motor points of triceps surae muscle. Int. J. Morphol., 30(3):105660, 2012.

Pai, C. H.; Lin, G. T.; Lin, S. Y.; Lin, S. D. \& Lai, C. S. Extensor digitorum brevis rotational muscle flap for lower leg and ankle coverage. J. Trauma, 49(6):1012-6, 2000.

Papalmitiades, M. Observations sur la fréquence d'un faisceau surnuméraire du muscle pédieux chez les Grecs. Bull. Mem. Soc. Anthropol. Paris, 9:177-9, 1948.

Riveros, A.; Sousa-Rodrigues, C. F. \& Olave, E. Origin and distribution of the motor branches for the superficial muscles of the anterior region of the forearm in Brazilian individuals. Int. J. Morphol., 34(1):356-64, 2016.

Rouvière, H. \& Delmas, A. Anatomía Humana. Vol. 3. 11 a ed. Barcelona, Masson, 2005.

Sarrafian, K. \& Kelikian, A. Sarrafian's Anatomy of the Foot and Ankle: Descriptive, Topographic, Functional. $3^{\text {a }}$ ed. Philadelphia, Lippincott Williams \& Wilkins, 2011.

Scott, A. T. \& Tuten, H. R. Calcaneonavicular coalition resection with extensor digitorum brevis interposition in adults. Foot Ankle Int., 28(8):890-5, 2007.

Sirasanagandla, S. R.; Swamy, R. S.; Nayak, S. B.; Somayaji, N. S.; Rao, M. K. \& Bhat, K. M. Analysis of the morphometry and variations in the extensor digitorum brevis muscle: an anatomic guide for muscle flap and tendon transfer surgical dissection. Anat. Cell Biol., 46(3):198202, 2013.

Standring, S. Gray's Anatomy. The Anatomical Basis of Clinical Practice. $41^{\mathrm{a}}$ ed. New York, Elsevier, 2016.

Testut, L. \& Latarjet, A. Tratado de Anatomía Humana. Vol. 3. $9^{\mathrm{a}}$ ed. Barcelona, Salvat, 1972.
Thompson, N. \& Gustavson, E. H. The use of neuromuscular free autografts with microneural anastomosis to restore elevation to the paralysed angle of the mouth in cases of unilateral facial paralysis. Chir. Plast., 3:16574, 1976.

Thompson, N. Treatment of Facial Paralysis by Free Skeletal Muscle Grafts. Londres, Fifth International Congress of Plastic and Reconstructive Surgery, 1971.

Tomaszewski, K. A.; Roy, J.; Vikse, J.; Pekala, P. A.; Kopacz, P. \& Henry, B. M. Prevalence of the accessory deep peroneal nerve: A cadaveric study and meta-analysis. Clin. Neurol. Neurosurg., 144:105-11, 2016.

Tzika, M.; Paraskevas, G. K. \& Kitsoulis, P. The accessory deep peroneal nerve: a review of the literature. Foot (Edinb.), 22(3):232-4, 2012.

Van Renterghem, D. \& De Ridder, K. Resection of calcaneonavicular bar with interposition of extensor digitorum brevis. A questionnaire review. Acta Orthop. Belg., 77(1):83-7, 2011.

Westlin, N. E.; Vogler, H. W.; Albertsson, M. P.; Arvidsson, T. \& Montgomery, F. Treatment of lateral ankle instability with transfer of the extensor digitorum brevis muscle. J. Foot Ankle Surg., 42(4):18392, 2003.

\author{
Dirección para correspondencia: \\ Dr. Enrique Olave \\ Facultad de Medicina \\ Universidad de La Frontera \\ Casilla 54-D \\ Temuco \\ CHILE
}

Email: enrique.olave@ufrontera.cl

Recibido : 06-01-2020

Aceptado: 18-03-2020 